\title{
Ultrasonic-assisted wound debridement for scleroderma digital ulcers
}

\author{
Antonella Marcoccia, ${ }^{1}$ Carlo Salvucci, ${ }^{1}$ Tina D'Alesio, ${ }^{1}$ Tarquinia Nuzzo, ${ }^{1}$ Anoush Vartanian, ${ }^{1}$ Tiziana Guastafierri, ${ }^{2}$ \\ Maria Grazia Modesti ${ }^{1}$ \\ ${ }^{1}$ Unit of Microvascular Diseases and Scleroderma Ulcers, Sandro Pertini Hospital; ${ }^{2}$ UOC of Clinical Biochemistry, Sandro Pertini \\ Hospital, Rome, Italy
}

\begin{abstract}
Debridement is a key element of wound care. It involves the removal of devitalized tissue from the wound bed in order to allow wound healing. Microcirculatory ulcers in general and scleroderma ulcers in particular are extremely painful, very reactive and rather unwilling to accept aggressive local treatments of a mechanical type or surgical cleaning which can promote an abnormal phlogistic response with a necrotizing evolution. For the elimination of devitalized tissue is necessary to promote autolytic or enzymatic debridement with slow tendency towards healing frequently exposed to phlogistic infections furthermore slowing healing with high risk of gangrene and amputation. Ultrasonic-assisted wound debridement uses low frequency ultrasound waves showing three clinical effects: atraumatic selective tissue debridement, wound stimulatory effect and antibacterial activity. We compare healing time, infections and procedural pain of ultrasonic debridement with autolytic debridement as for Scleroderma digital Ulcers. There is evidence to show that Ultrasonicassisted wound debridement reduces the time of healing, needing for antimicrobial therapy, the procedural pain and consequently the cost to the healthcare system improving the patients' quality of life.
\end{abstract}

\section{INTRODUCTION}

Debridement means removing dead, damaged or infected tissue from wound in order to increase the healing potential of remaining healthy tissue. ${ }^{1-3}$ There are several methods of debridement and that is essential for a good preparation: surgical technique, high pressure irrigating

Correspondence: Antonella Marcoccia, Unit of Microvascular Diseases and Scleroderma Ulcers, Sandro Pertini Hospital. Via dei Monti Tiburtini 385, 00157 Rome, Italy

Tel.: +39.06.41433585.

E-mail: antonella.marcoccia@aslroma2.it

Key words: wound care, debridement, scleroderma digital ulcers.

Contributions: AM recruited the patients, designed the research and wrote the paper; CS managed the wound care and debridement; TD, TN, AV collected laboratory and clinical data. AM, TG, MGD analysed and discussed the data.

All the authors read and approved the final manuscript.

Conflict of interest: the authors declare no potential conflict of interest.

Received for publication: 9 May 2017.

Revision received: 21 September 2017.

Accepted for publication: 3 November 2017.

This work is licensed under a Creative Commons Attribution NonCommercial 4.0 License (CC BY-NC 4.0).

(C) Copyright A. Marcoccia et al., 2017

Licensee PAGEPress, Italy

Italian Journal of Wound Care 2017; 1(2):73-78

doi:10.4081/ijwc.2017.11 device, chemical debridement, enzymatic preparation, autolytic hydrogels and hydrocolloids, the old larva therapy and the more recent negative pressure therapy. Ultrasonicassisted wound debridement is a less known debridement method that uses slow frequency ultrasound waves. ${ }^{3,4}$

Scleroderma digital ulcers (DU) are typically acral ischemic lesions of the fingers and toes with a recurring chronic course. They are the results of spasms and occlusions of the arterial-capillary region of the fingers, determining ischemic and hypoxic damage presenting like a localized hyperkeratosis on the distal phalanges up to dermohypodermic-muscular necrosis and acroosteolysis. Microcirculatory ulcers in general and scleroderma ulcers in particular are extremely painful, very reactive and rather unwilling to accept local aggressive treatments of mechanical type or surgical cleaning as this can promote abnormal inflammatory reaction with a necrotizing evolution. Therefore, the removal of devitalized tissue is done by preferring the autolytic or enzymatic debridement (enzymatic preparation, autolytic hydrogels and hydrocolloids) characterized by repeated dressings and a slow healing process that takes several months. ${ }^{3}$ Because of their slow tendency towards healing and long duration DU are frequently exposed to infective inflammatory complications that further slow healing.

Sometimes, in order to avoid procedural pain, Scleroderma patients live for several months with digital necrotic eschar exposed to any treatment. The long period of traditional non traumatic debridement or the failure of any debridement can expose the patient to the risk of acute infectious inflammatory complications which may result in necrotizing evolutions until the dead end of gangrene and amputation ${ }^{5}$ (Figure 1).

Therapies for nonhealing wounds have mostly focused 
on correcting factors involved in chronic wounds. Over the last 25-30 years, a spectrum of advanced therapeutic approaches has evolved while even basic wound care has continued to improve. These therapies include antibiotic treatment of cellulitis, removal of possible biofilms, revascularization of ischemic limbs, offloading of decubitus (pressure) ulcers, negative pressure to remove fluid, Advanced treatments for difficult wounds are needed. Recently stem cell therapy has emerged as a new approach to accelerate wound healing: autologous adipose tissue derived cells in open label studies accelerate wound healing in scleroderma digital ulcers ${ }^{6,7}$ However, there are some potential limitations to successful stem cell therapy including suboptimal debridement for wound bed preparation. ${ }^{8}$ Appropriate ulcer care principles also include optimizing the wound bed by debridement, facilitating reduction of edema, decreasing bacterial burden, and providing the right balance of moisture. ${ }^{8}$

Ultrasonic-assisted wound debridement is a lesser known debridement method that uses low frequency ul- trasound waves. Scientific literature highlights the main characteristics of Ultrasonic-assisted debridement as an atraumatic surgical debridement and painless compared to killing bacterial action resulting in reduction of microbial load induced by temperature and by the process of cavitation and selectivity that protects healthy tissue. The ultrasound device (Figure 2) Surgysonic Wound ( Esacrom consists of a console with a peristaltic pump (for irrigation, disinfection and cooling of the site to be treated) plus a piezoelectric ultrasonic hand-piece designed to fragment and remove necrotic tissues, a metal rod to maintain the fluid reservoir, a metal rod for the hand piece, a pneumatic pedal and various types of tips, each for a different form of lesion. It is used in continuous and pulsed mode to optimize performance, according to the sensitivity of the patient and the type of tissue involved.

It generates ultrasounds able to trigger the tip used for removal of tissue, as well as to control the temperature of the tip using saline previously cooled to about $4{ }^{\circ} \mathrm{C}$, in direct contact with the treated lesion. Ultrasounds are waves
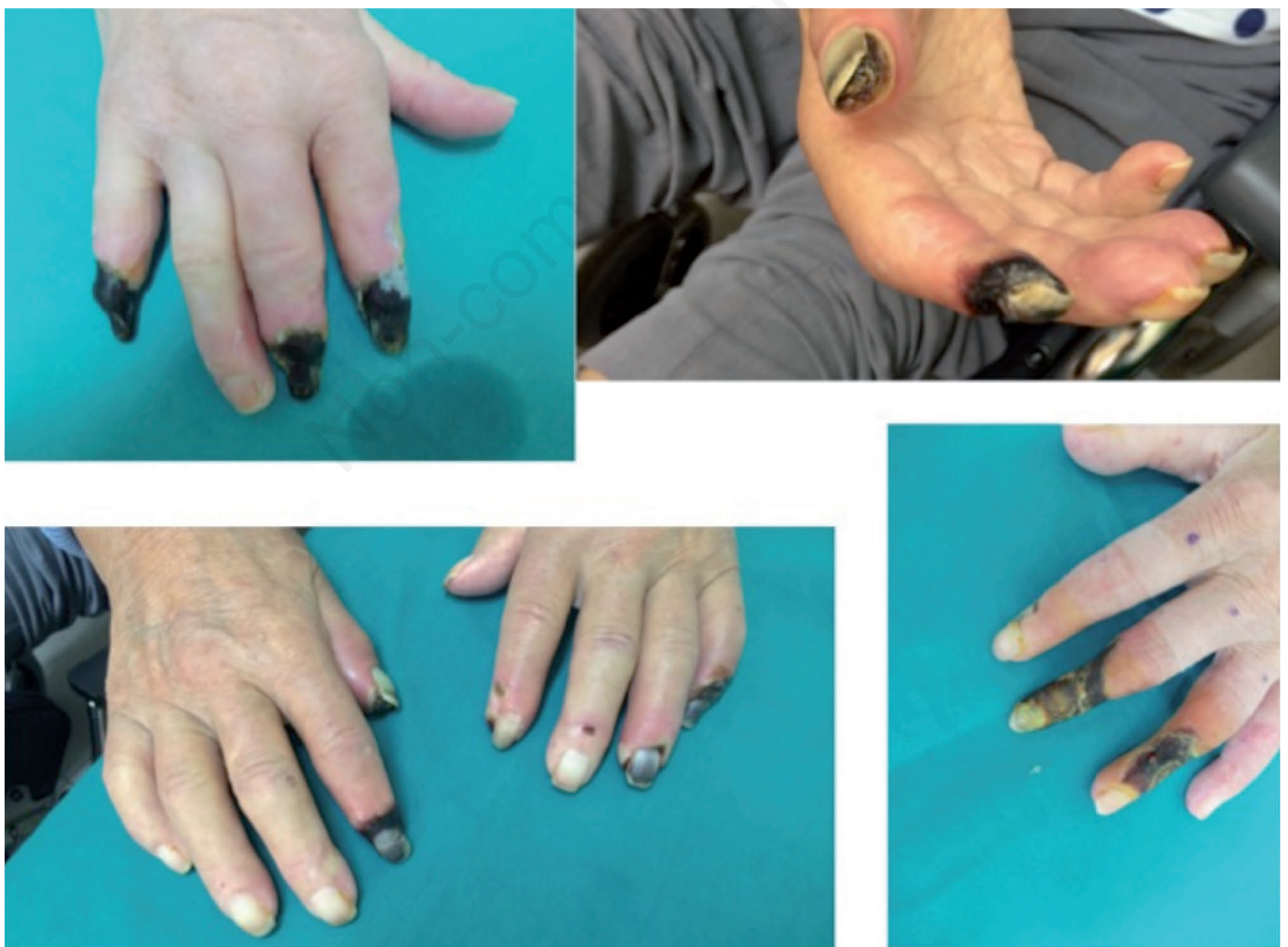

Figure 1. Scleroderma gangrene. 
of higher frequency than what perceived by the ear allowing the vibration of the hand-piece of the instrument at a frequency of about $30.000 \mathrm{~Hz}$ per second. The basic process involves the cavitation of water drops by emitting ultrasounds: this occurs when a liquid is subjected to ultrasounds in such a way that the acoustic wave causes the formation, growth and rapid decompression of steam bubbles in the liquid, forming areas of vapor within the liquid which subsequently collapse and implode. This is due to the local decrease of pressure to a lower value than the vapor pressure of the liquid itself, which becomes a gas and forms cavities containing vapor. Acceleration of the insert due to vibration and emission of sequence liquid saline phases generate pressure and vacuum. This forms microbubbles containing vapor that solicited from external pressure implodes exerting pressure on surrounding microbubbles

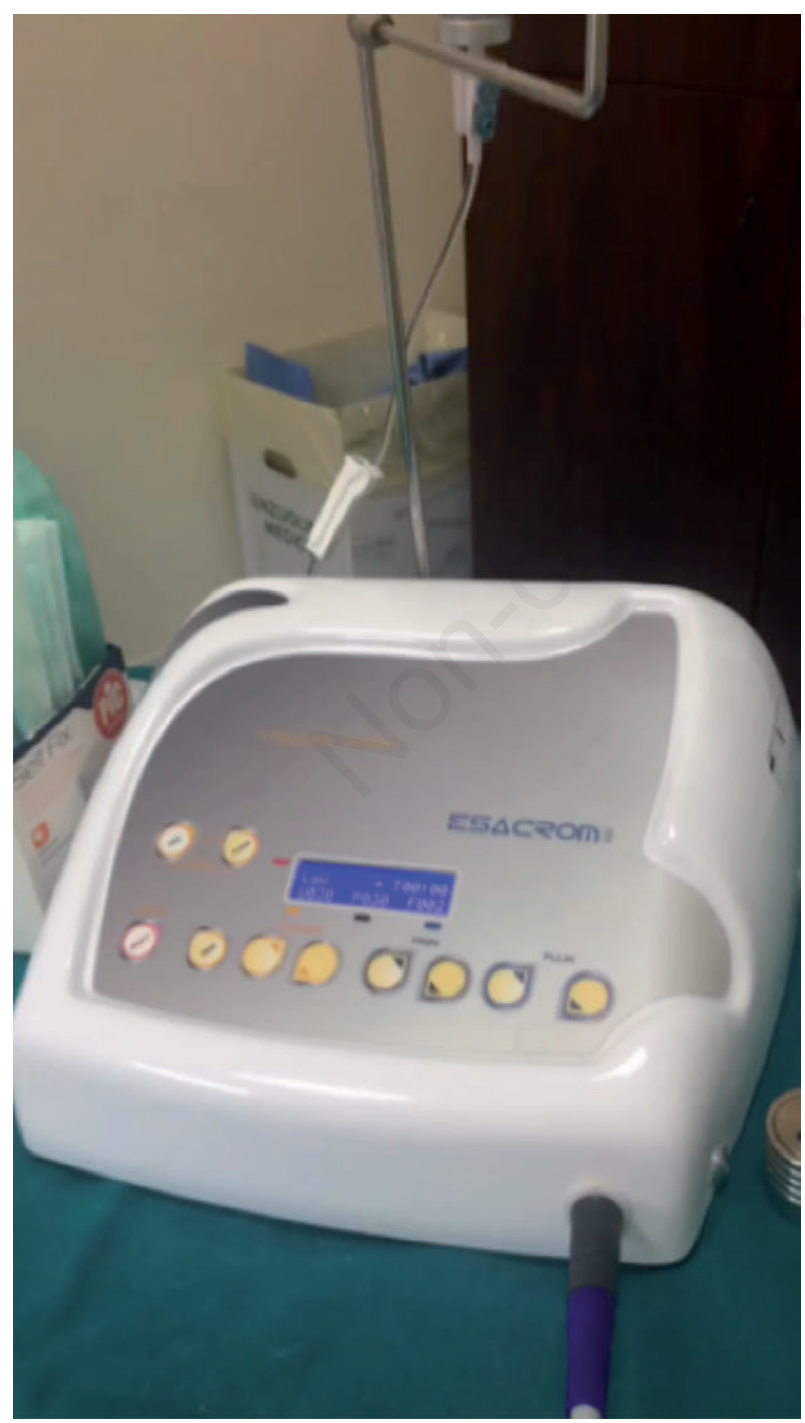

Figure 2. Surgysonic Wound ${ }^{\circledR}$-Esacrom. which in turn favors cavitation, dissection of tissues at different densities, fragmentation of fibrin and necrotic tissue and finally debridement. This mechanism detaches tissues of different densities, to emulsify the fibrin or biofilm and thus eliminate necrosis. Thanks to its elastic properties, healthy tissue owns higher vibration amplitude and deformation capacity and therefore is preserved. ${ }^{9,10}$

Technique is relatively simple, results are immediate and selective, so it does not affect healthy tissue and causes no pain throughout the process of removing the excess material. This makes the technique particularly suitable for scleroderma ulcers treatment. Compared to classic debridement such as enzyme preparations (which require frequent applications with the risk of skin sensitization and the onset of burning sensations in addition to having a slow action especially on thick necrotic layers) and autolytic debridement (in which dressing stimulates the autonomous production of autolytic enzymes by modifying the environment at the basis of the lesion, yielding/absorbing moisture and gelling causing frequent maceration of the edges and acting very slowly), Ultrasound-assisted debridement is proposed as an advanced technique complementary to traditional debridement in the treatment of scleroderma ulcers for cleaning the wound bed of the lesion in an atraumatic and painless way.

\section{MATERIALS AND METHODS}

We conducted a case-control study of patients referred to our department for scleroderma ischemic digital ulcers from September 2016 to December 2016. We compared 5 patients treated with ultrasound technique (Surgysonic Wound $(\mathrm{B}$-Esacrom) referred as A Group with the clinical course of 5 patients with scleroderma digital ulcers with similar characteristics but treated exclusively with traditional debridement (Hydrogels) B Group. We compared as outcomes the time of healing (days), the need for antimicrobial therapy, the pain perception on a visual analogue scale (VAS Scale 0-10) during dressing as procedural pain.

All the treated ulcers had a chronic course with an onset $>8$ weeks and presented a necrotic eschar $\leq 2 \mathrm{~cm}$ size distal to the proximal interphalangeal joint on the fingertips, on the volar aspect of the fingers or on the nail base without radiological bone involvement (without radiographic features of osteomyelitis). In order to prevent or reduce the onset of pain treating ulcers, a local anesthetic ointment with lidocaine 5\% was applied 15 minutes before dressing. ${ }^{11}$ Dressing's frequency was twice a week.

A Group: in the first session of debridement the number of ultrasonic applications ranged from 3 to 6 with an average time of 50-60 seconds and up to a total time of maximum 6 minutes application obtaining the progressive excision of the necrotic eschar. From the second treatment we observed an initial granulation process and each time 
we did individual applications of 40-50 seconds, sufficient for the removal of the bottom bound. At the end of each session of debridement, hydrogel and paraffin gauze were applied on the lesion.

B Group: in the first dressing hydrogel and paraffin gauze were applied on the necrotic eschar From the second dressing we tried to remove the devitalized tissue and the previous dressing's remnants using saline solution, gauze and only the needle tip 16 gauge without touching healthy periwound tissue ( $>5$ VAS).

\section{Statistic analysis}

Clinical features of patients were analyzed using the Student's t-test for unpaired data. Meaningful comparisons were considered when $\mathrm{P}<0.05$ (Table 1).

\section{RESULTS}

A Group: no patients needed antibiotic therapy throughout the treatment period and all patients showed good compliance of the therapy and referred a mild and bearable pain symptoms $\leq 3(2.2 \pm 0.8)$ according to the VAS Scale. Healing occurred after a treatment of 4-8 weeks with an average of $30.6 \pm 21.1$ days (Figure 3).

B Group: 1 patient showed infections and phlogistic signs and underwent targeted antibiotic therapy. At every access to medication patients reported a pain $>5(7.0 \pm 1.2)$ according to the VAS Scale. After 8 weeks the lesions were still at the stage of debridement with initial signs of granulation and were healed in an average time of $75 \pm 13.1$ days (Figure 4).

\section{DISCUSSION}

From June 2016 we started to use ultrasound debridement and we treated a very heterogeneous population of scleroderma patients with digital ulcers using ultrasound debridement and we observed from the first time a good compliance and a strongly favorable outcome in all patients, suggesting to analize and quantify the different outcome between traditional autolytic debridement and ultrasound debridement, comparing two groups of patients with the similar size and positions of scleroderma ulcers, same subset of disease, same systemic therapies, same seasonal enrollment period, same beginning of treatment, same operator, in order to support our observation and experience.

The strong favorable outcome regarding the time of healing, needing for antimicrobial therapy, the procedural pain has led us to use ultrasound debridement for the benefit of all patients and to limit the case-control study to 10 patients ( 5 case and 5 controls). Although the small size of this study, ultrasound debridement is promising in the treatment of scleroderma ulcers and need to confirm the preliminary evidences with other studies.

\section{CONCLUSIONS}

Ultrasound-assisted debridement, not recently introduced, has been revived thanks to the production of the industry of medical equipment with new models capable of offering the most sensitive and selective debridement procedures. It has long been used as a dental technique for tartar removal and fort other local interventions. Scientific literature underlines the main characteristics that favor ultrasonic debridement in wound care. It is atraumatic and painless due to the ability of selectivity that protects healthy tissue. It also has a killing bacterial activity, resulting in reduction of microbial load induced by temperature and cavitation processes. We compare healing time, infections rate and procedural pain of ultrasonic debridement with traditional autolytic debridement for Scleroderma digital Ulcers. We reported that Ultrasound

Table 1. Clinical features, systemic therapy of digital ulcers and pain.

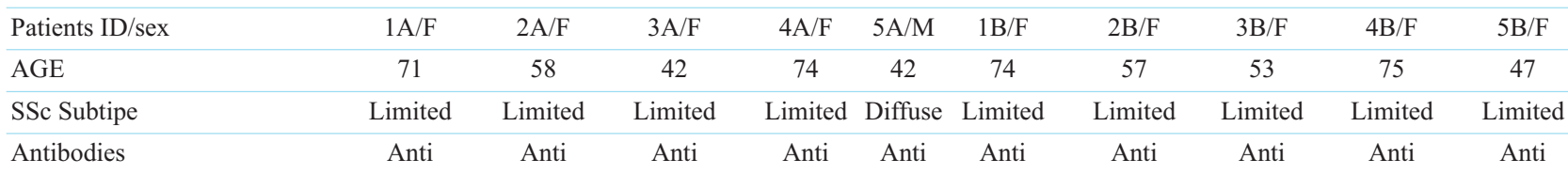

Antibodies

centromere centromere centromere centromere scl70 centromere centromere centromere centromere centromere

\begin{tabular}{|c|c|c|c|c|c|c|c|c|c|c|}
\hline Smoker & no & no & no & no & no & no & no & no & no & no \\
\hline $\begin{array}{l}\text { Iloprost } 5 \text { days/mont: } \\
2 \mathrm{ng} / \mathrm{kg} / \mathrm{min} 6 \mathrm{~h} / \text { day }\end{array}$ & yes & yes & yes & yes & yes & yes & yes & yes & yes & yes \\
\hline Bosentan $125 \mathrm{mg} \times 2$ & yes & yes & yes & yes & yes & yes & yes & yes & yes & yes \\
\hline Antiaggregation ASA $100 \mathrm{mg}$ & yes & yes & yes & yes & yes & yes & yes & yes & yes & yes \\
\hline Calcium channel blockers & yes & yes & yes & yes & yes & yes & yes & yes & yes & yes \\
\hline Acetaminophen+contramal & yes & yes & yes & yes & yes & yes & yes & yes & yes & yes \\
\hline
\end{tabular}



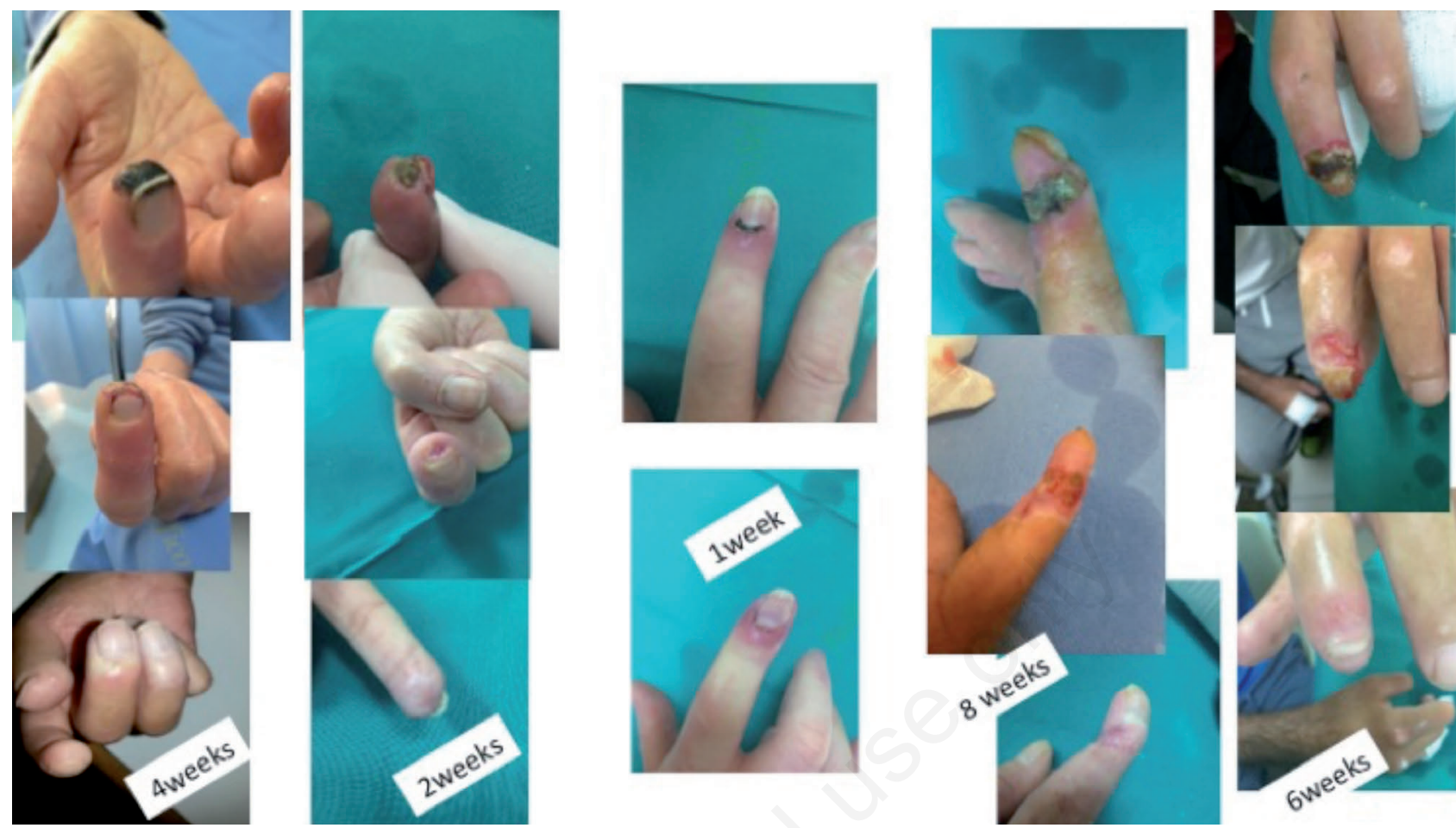

Figure 3. A Group: ultrasound debridement.
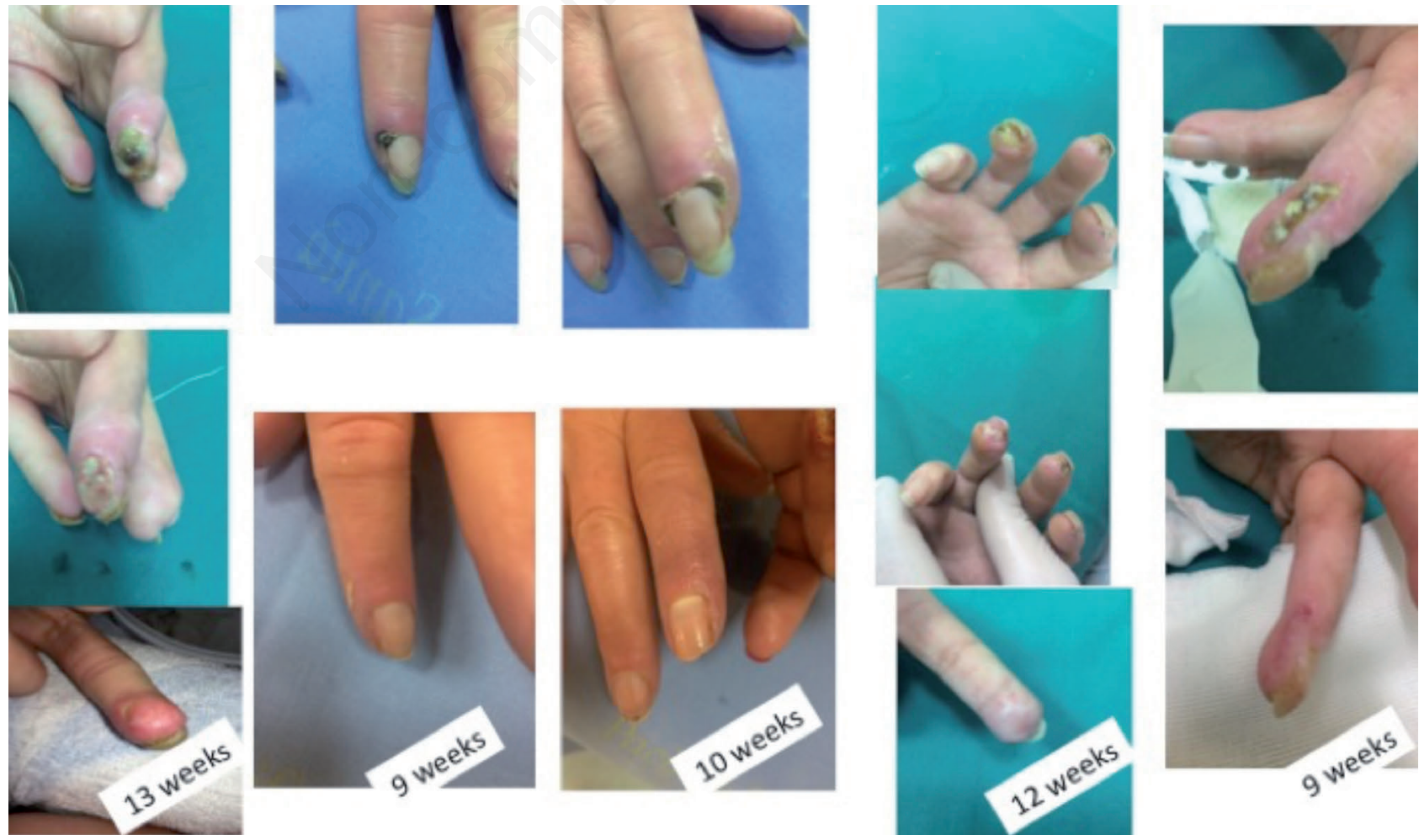

Figure 4. B Group: autolitic debridement. 
debridement leads to a significant reduction in healing time (P-value 0.004) (Figure 5) and the rate of infections and therefore presents a good patient compliance to treatment thanks to a significant reduction of procedural pain (P-value 0.001) (Figure 6).

TIME OF HEALING

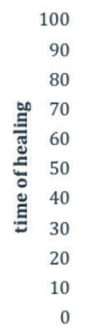

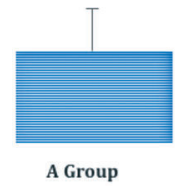

vs

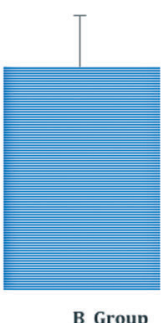

Figure 5. Time of healing. A Group (ultrasonic debridement) $v s$ B Group (autolitic debridement): $30.6 \pm 21.1$ vs $75 \pm 13.1$ days, Pvalue 0.004 .

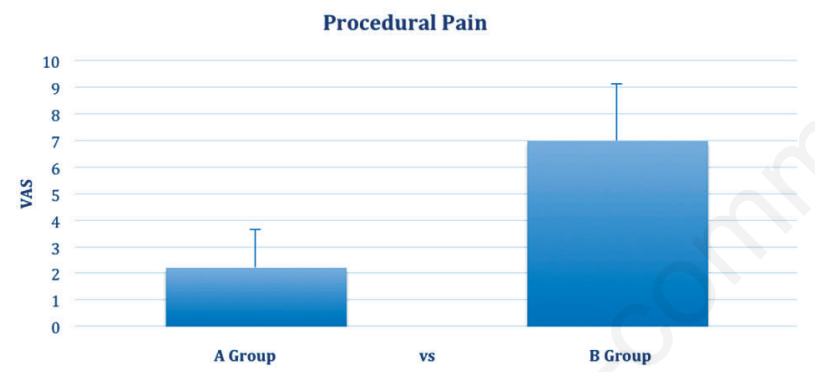

Figure 6. Procedural pain VAS scale. A Group vs B Group: $2.2 \pm 0.8$ vs $7.0 \pm 1.2$, P-value 0.001 .

\section{REFERENCES}

1. Wolcott RD, Rhoads DD, Bentt ME et al. Chronic wounds and medical biofilm paradigm. J Wound Care 2010;19:45-53.

2. Ayello EA, Cuddigan JE. Debridement: controlling the necrotic/cellular burden. Adv Skin Wound Care. 2004;17: 66-75.

3. Strohal R, Dissemond J, Jordan O et al. An updated overview and clarification of the principle role of debridement. J Wond Care 2013 J;22:S1-S52.

4. Nichter LS, Milliams J. Ultrasonic wound debridement. J Hand Surg Am 1988;13:142-6.

5. Mollo PE, Di Salvo MM, Failla G et al. Documento posizionamento ulcera ischemiche. Associazione Italiana Ulcere Cutanee (AIUC). Acta Vulnol 2012;10.

6. Bene MD, Pozzi MR, Rovati L et al. Autologous fat grafting for scleroderma induced digital ulcers. An effective technique in patients with systemic sclerosis. Handchir Mikrochir Plast Chir 2014;46:242-7.

7. Del papa N, Di Luca G, Sambataro D et al. Regional implantation of autologous adipose tissue derived cells induces a prompt healing of long lasting indolent digital ulcers in patients with systemic sclerosis. Cell Transplant 2015;24: 2297-305.

8. Vinas MO, Falanga V. Mesenchymal stem cells in chronic wounds: the spectrum from basic to advanced therapy. Adv Wound Care (New Rochelle) 2016; 5:149-63.

9. Voigt J, Wendelken M, Driver V, Alvarez OM. Low-frequency ultrasound $(20-40 \mathrm{kHz})$ as an adjunctive therapy for chronic wound healing: a systematic review of the literature and meta-analysis of eight randomized controlled trials. Int J Low Extrem Wounds 2011;1:190-9.

10. Butcher G, Pinnuck L. Wound bed preparation: ultrasonic assisted debridement. Br J Nurs 2013;22:38-43.

11. Giacinto F. Application of ortodermina ointment (lidocaine $5 \%$ ) when preparing ulcers for ultrasonic debridement. Acta Vulnol 2016;14:16-23. 